220 patients from 8 studies, allowed a random effects model meta-analysis to be carried out. The mean age of patients within individual studies ranged between 45-61 years. 64\% of patients were female. The underlying primary malignancy was gastrointestinal (53\%), gynaecological (25\%) (accounting for the female predominance), and others (22\%). Four studies reported use of palliative chemoradiotherapy with $39-100 \%$ of the patients under going at least one cycle of treatment. Figure 1 shows the survival at monthly intervals for six months. The overall median survival is 83 days. $24 \%$ were still alive at 6 months but only about $2 \%$ at year.

Conclusion This is first systematic review showing the survival in patients with malignant inoperable bowel obstruction receiving HPN during the palliative phase of care. We show, using the largest published cohort to date, that the median survival is only 83 days. The described variability in survival length between studies and between patients can be substantial. This information can help inform clinician decisions about the use of HPN in such patients.

Disclosure of Interest None Declared.

\section{OC-026 DIAGNOSTIC YIELDS OF SEHCAT SCANNING AND GLUCOSE HYDROGEN BREATH TESTING IN PELVIC RADIATION DISEASE}

R Stacey*, J Turner, J Green. Department of Gastroenterology, University Hospital Llandough, Cardiff, UK

\subsection{6/gutjnl-2014-307263.26}

Introduction Late Gastrointestinal side effects following radiotherapy are common but under reported by patients and poorly recognised by clinicians ${ }^{-1}$ Pelvic radiation disease (PRD) can present up to 30 years after radiotherapy treatment with a range of symptoms, including rectal bleeding, abdominal pain and diarrhoea. Many of these patients experience troublesome symptoms of diarrhoea secondary to Bile acid Malabsorption (BAM) and small intestinal bacterial overgrowth (SIBO).

In our centre we have access to 23-seleno-25-homo-taurocholic acid (SeHCAT) nuclear medicine scanning and glucose hydrogen breath testing for the investigation of BAM and SIBO associated with PRD. The majority of data on patients with pelvic radiation disease has been published by one specialist centre (The Royal Marsden Hospital). We audited the diagnostic yields for investigation of small intestinal manifestations of PRD.

Methods We identified 77 patients referred to gastroenterology clinic at University Hospital Llandough, Cardiff with suspected pelvic radiation disease via a departmental database. All patients were investigated via the Royal Marsden algorithm and BSG guidance. We then identified all patients with diarrhoeal symptoms and audited the results of any SeHCAT and glucose hydrogen breath tests and their diagnostic yields.

Results 26 patients (36\%) had diarrhoeal symptoms. Within this group, 24 patients underwent SeHCAT scanning, with $45 \%$ having positive results. 19 were referred for glucose hydrogen breath tests of which $53 \%$ had positive results. $17 \%$ had both positive SeHCAT and Glucose hydrogen breath tests. Not all patients received both tests due to various reasons (clinical decision, patient choice and non attendance).

Conclusion PRD is common but under investigated. BSG guidance on its management exists ${ }^{[1]}$ and an algorithmic approach has been shown to be beneficial. ${ }^{2}$ Diarrhoea occurs frequently in
PRD, and we have found that SeHCAT scanning and Glucose Hydrogen breath tests in these patients have a high diagnostic yield for BAM and SIBO respectively. SIBO and BAM can exist concurrently in this group, and our data supports a thorough and systematic algorithmic approach. Clinicians should have a low threshold for requesting these tests in patients with suspected PRD.

\section{REFERENCES}

1 Andreyev HJN, Davidson SE, Gillespie C, Allum WH, Swarbrick E. British Society of Gastroenterology, et al. Practice guidance on the management of acute and chronic gastrointestinal problems arising as a result of treatment for cancer. Gut 2012:179-92

2 Andreyev HJN, Benton BE, Lalji A, Norton PC, Kabir M, Gage PH, et al. ArticlesAlgorithm-based management of patients with gastrointestinal symptoms in patients after pelvic radiation treatment (ORBIT): a randomised controlled trial. Lancet. Elsevier Ltd; 2013 Sep 20:1-9

Disclosure of Interest None Declared.

\section{OC-027 FGF19 LEVELS IN SUBJECTS WITH PRIMARY BILE ACID DIARRHOEA AND ELEVATED TRIGLYCERIDES}

${ }^{1} \mathrm{~J}$ Nolan, ${ }^{2} \mathrm{~S}$ Pattni, ${ }^{1} \mathrm{JR}$ Walters, IM Johnston*. 'Imperial College London, London, UK; ${ }^{2}$ University of Leicester, Leicester, UK

10.1136/gutjnl-2014-307263.27

Introduction There is evidence that primary bile acid diarrhoea (PBAD) is caused by disordered bile acid homeostasis. Most patients with severe PBAD have low fasting serum FGF19 which fails to rise above $300 \mathrm{pg} / \mathrm{ml}$ postprandially. Different patterns of postprandial FGF19 response have been demonstrated, with some resembling those of healthy individuals. Others have shown that serum triglyceride levels reflect expression of the luminal bile acid transporter ASBT. It is hypothesised that a subset of individuals with hypertriglyceridaemia have different fasting FGF19 levels and postprandial FGF19 response.

Methods Study 1: 162 patients with chronic diarrhoea were recruited prospectively. All patients underwent routine testing to exclude other causes of diarrhoea and had SeHCAT tests. Patients were classified as having PBAD, or unexplained chronic diarrhoea (CD). Other diagnoses were excluded. Fasting blood samples were taken and processed for triglycerides and FGF19. Subjects with either diagnosis were also analysed within 2 subgroups according to triglyceride level (cut off $2.30 \mathrm{mmol} / \mathrm{l})$. Study 2: 18 subjects took part in a study to examine FGF19 levels over the course of $6 \mathrm{~h}$. After an overnight fast, blood was sampled every $90 \mathrm{~min}$ for $6 \mathrm{~h}$. Meals were provided at 9 am and 12 noon. Serum FGF19 was quantified by ELISA using a commercially available kit. Triglycerides were quantified by standard colorimetric technique. Mann-Whitney and Spearman rank correlation tests were used in analyses.

Results Study 1: Overall subjects with elevated triglycerides $(\mathrm{n}=18)$ have significantly lower SeHCAT retention (median 7.95 vs. $19.5 \% \mathrm{p}=0.01$ ). Subjects with severe BAD with elevated triglycerides had higher fasting FGF19 levels (241 vs $101 \mathrm{pg} / \mathrm{l} \mathrm{p}=0.02$ ). Study 2: There was no significant difference in fasting triglycerides between different phenotypes of FGF19 response (previously presented work). The percentage increase in FGF19 from fasting to $90 \mathrm{~min}$ after breakfast correlates with fasting serum triglyceride level $(\mathrm{R}=0.59, \mathrm{p}<0.005)$. 
Conclusion We have identified a subset of PBAD subjects with high triglycerides and fasting FGF19 levels comparable to healthy individuals. The post prandial rise in FGF19 suggests no defect in the response of FGF19 synthesis in this subset. It may instead be caused by impaired BA absorption due to reduced ASBT expression which is also manifested as high serum triglycerides. PBAD may be a heterogenous condition with more than one underlying key abnormality.

Disclosure of Interest None Declared.

\section{OC-028 SMALL BOWEL CANCER IN THE UK}

${ }^{1} \mathrm{~L}$ Chan*, ${ }^{2} \mathrm{G}$ Evans. 'Gastroenterology, Manchester Royal Infirmary, Manchester, UK; ${ }^{2}$ Genetic Medicine, Manchester Royal Infirmary, Manchester, UK

\subsection{6/gutinl-2014-307263.28}

Introduction Small bowel cancer (SBC) is uncommon worldwide and accounts for only 5\% of all gastrointestinal (GI) malignancies despite the small bowel forming $75 \%$ of the GI tract. [1] Our understanding is limited by its rarity, insidious course, difficult assessment and late diagnosis, coupled with multiple histological subtypes. We aim to review the trend of SBC in the UK over the last two decades.

Methods The national UK Association of Cancer Registries (UKACR) database identified SBC patients diagnosed from January 1991 to January 2009. We retrospectively reviewed and carried out a descriptive analysis of SBC incidence rates with respect to gender, age, ethnicity (as per UK Census 2001) and socio-economic status (as per UK Indices of Deprivation 2004, 2007 and 2010) and mortality rates.

Results The registry identified 11,872 patients, 53.6\% male and $46.4 \%$ female, who were diagnosed at a singular peak mean age of 67 years over the study period. The overall incidence of SBC increased from 0.71 to 1.51 per 100,000 from 1991 to 2009 with mortality increasing simultaneously but to a lesser extent (Figure 1A). SBC was 1.5 times more common in males than females. They were most frequently located at the duodenum $(57.5 \%, \mathrm{n}=7860)$ where incidence almost tripled $(0.24$ to 0.63 per 100,000), and less frequently at the jejunum (12.1\%) and ileum (30.4\%) where incidence approximately doubled (0.07 to 0.11 and 0.14 to 0.33 per 100,000 respectively). The incidence in white patients was 1.5 times higher than black patients and 3 times higher than in Asian patients over the period 2001 to 2009 (Figure 1B). SBC incidence was unchanged with respect to socio-economic status.
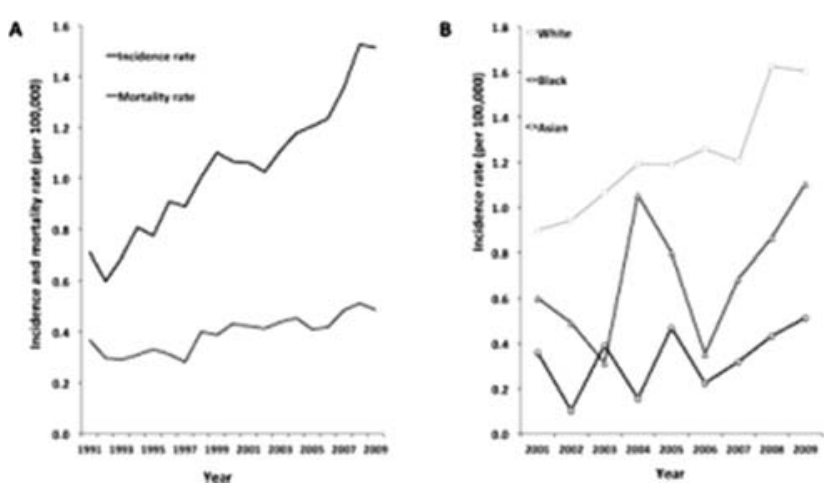

Abstract OC-028 Figure 1 (A) SBC incidence and mortality rates, 1991 to 2009. (B) SBC incidence by ethnic group, 2001 to 2009
Conclusion The incidence of SBC in the UK has increased over the last two decades with little improvement in mortality rates. It is most common in males in their $6^{\text {th }}$ decade and in the proximal small intestine, which is in keeping with current literature. However, the higher incidence in white patients is in contrast to the geographical variation seen in both United States SBC and UK colorectal cancer data. A more comprehensive understanding of the natural history, environmental and genetic predisposition is needed to allow for potential patient stratification, more efficient diagnosis and treatment and thus improving its poor prognosis.

\section{REFERENCE}

1 Ross et al. British Journal of Cancer. 1991;63:143-145

Disclosure of Interest None Declared.

\section{Liver section free papers}

\section{OC-029 RIFAXIMIN IS EFFICACIOUS IN THE TREATMENT OF CHRONIC OVERT HEPATIC ENCEPHALOPATHY: A UK LIVER MULTI-CENTRE EXPERIENCE}

${ }^{1} \mathrm{VC}$ Patel ${ }^{*},{ }^{2} \mathrm{~J}$ Orr, ${ }^{1} \mathrm{~J}$ Sturgeon, ${ }^{1} \mathrm{Z}$ Habtemariam, ${ }^{3} \mathrm{H}$ Preedy, ${ }^{4} \mathrm{P}$ Richardson, ${ }^{3} \mathrm{R}$ Aspinall, ${ }^{2} \mathrm{M}$ Hudson, 'DL Shawcross. 'Institute of Liver Studies, King's College Hospital NHS Foundation Trust, London, UK; ${ }^{2}$ Department of Hepatology, Freeman Hospital, Newcastle Hospitals NHS Foundation Trust, Newcastle, UK; ${ }^{3}$ Department of Gastroenterology and Hepatology, Queen Alexandra Hospital, Portsmouth Hospitals NHS Trust, Portsmouth, UK; ${ }^{4}$ Department of Hepatology, Royal Liverpool University Hospital, Liverpool, UK

\subsection{6/gutjnl-2014-307263.29}

Introduction Rifaximin- $\alpha$ is a non-absorbable antibiotic increasingly being used for the secondary prevention of recurrent overt hepatic encephalopathy (HE) in the UK. The therapeutic mechanism of rifaximin has yet to be elucidated, with reduction in gut ammonia production postulated. We undertook a UK multicentre retrospective audit of patients receiving rifaximin therapy for HE in 4 hospitals, two of which are liver transplant units, with the aim of assessing tolerability, impact on HE/liver disease severity and hospitalisation rates.

Methods Patient demographics, concurrent therapy, Child Pugh, MELD, UKELD and number of hospital admissions were collected 3 months prior to initiation of rifaximin therapy and then 3 months following treatment.

Results 170 patients were identified (mean age 57yrs \pm 12 ; 68\% male) over the period 05/2010-03/2013. Three month post treatment outcome data were available for 73 patients (43\%); 53 patients (31\%) died during the 3 month follow up period. Average duration of treatment was $79 \pm 121$ days, with therapy well tolerated in $97.6 \%$ of patients. $74 \%$ were taking concomitant lactulose with $23.5 \%$ on rifaximin monotherapy. No cases of Clostridium difficile infection were reported.

The most common aetiology was alcohol 90/170 (53\%) with $25(28 \%)$ actively drinking . 36 patients (21\%) were transplanted during the audit period. The predominant HE phenotype was episodic overt (67\%), with persistent overt featuring in $20 \%$ and the Parkinsonian phenotype in $6 \%$.

Admission data were available for $143 / 170$ (84\%) patients with a total of 444 admissions in the 3 months prior to therapy (average admission length $23 \pm 25$ days). The hospitalisation rate per patient fell significantly from $2.7 \pm 3.2$ to $1.0 \pm 1.8$ admissions in the 3 months following initiation of therapy $(\mathrm{p}<$ 